\title{
The Effects of Sleep Deprivation on the Biophysical Properties of Facial Skin
}

\author{
Min Ah Kim, Eun Joo Kim, Byung Young Kang, Hae Kwang Lee* \\ Skincare Research Institute, AMOREPACIFIC R \& D Center, Yongin, Korea \\ Email: ^haekwang@amorepacific.com
}

How to cite this paper: Kim, M.A., Kim, E.J., Kang, B.Y. and Lee, H.K. (2017) The Effects of Sleep Deprivation on the Biophysical Properties of Facial Skin. Journal of Cosmetics, Dermatological Sciences and Applications, 7, 34-47.

https://doi.org/10.4236/jcdsa.2017.71004

Received: January 25, 2017

Accepted: March 4, 2017

Published: March 7, 2017

Copyright $(9) 2017$ by authors and Scientific Research Publishing Inc. This work is licensed under the Creative Commons Attribution International License (CC BY 4.0).

http://creativecommons.org/licenses/by/4.0/

\begin{abstract}
Lack of sleep is a problem in today's society, and many people are concerned about changes in their outward appearance due to lack of sleep. People generally come up with some noticeable skin attributes as symptoms of sleep deficiency including rough, dull, and dry skin as well as droopy eyelids and dark eye circles. Several previous reports also suggested that poor sleep could affect the skin condition. The purpose of this study was to evaluate the effect of one night of sleep deprivation on various skin biophysical properties. Twenty four healthy females participated in a study of one night of sleep deprivation. Subjects were kept awake for one night in a laboratory with controlled temperature and relative humidity. The skin condition of each subject was evaluated after normal sleep pattern and after one night of sleep deprivation. The measured skin biophysical parameters included transepidermal water loss, facial pore size, and skin tone, hydration, elasticity, desquamation, translucency, and blood flow. The cheek, eye, and lip areas were evaluated. After one night of sleep deprivation, multiple skin biophysical parameters showed changes when compared to the baseline measurements. A significant decrease in skin hydration and impaired barrier function were observed $(\mathrm{p}<0.05)$. Decreased hydration led to decreased skin elasticity and translucency and increased skin scaling $(\mathrm{p}<0.05)$. Facial pores were more conspicuous, and skin lightness decreased significantly $(\mathrm{p}<0.05)$. Furthermore, skin blood flow decreased prominently $(\mathrm{p}<0.05)$. The results demonstrated that the skin features that are recognized as symptoms of sleep deficiency actually showed remarkable differences after a period of sleep deprivation, and some of these features were confirmed in the eye, lip, as well as the cheek areas. This study revealed a significant association between sleep deprivation and skin biophysical properties by scientific measuring.
\end{abstract}

\section{Keywords}

Sleep Deprivation, Skin Biophysical Property, Hydration, Skin Barrier 


\section{Introduction}

Sleep is vital to human physiological, cognitive, and behavioral functions [1] [2] [3] [4], as well as long term health [4] [5]. It is a recurrent state of bodily rest and reduced consciousness that serves multiple functions, which is associated to the circadian system [6] [7]. Lack of sleep is a major issue in society today, causing many people to suffer from health problems related to sleep deprivation [5] [8]. Furthermore, many people are concerned about changes in their outward appearance due to lack of sleep [9].

The skin is the largest organ in the human body and plays a crucial role in maintaining homeostasis. Several reports suggest that poor sleep alters the skin condition. One study [10] found the scientific clues of the common expression "beauty sleep", investigating a correlation between an individual's state of sleep and their attractiveness. Sleep deprived people were rated as less healthy and less attractive than after a normal sleep condition by visual scoring. Another study of visual assessment reported that the eyes of sleep deprived individuals were perceived as redder, more swollen, having darker circles under the eyes, and the skin as paler, having more wrinkles and fine lines after 31 hours of sleep deprivation [9]. These facial regions, which are affected by sleep deprivation, are important for social communication between people [9] [11]. Therefore, it is unsurprising that people come up with some noticeable skin attributes as symptoms of sleep deficiency. Such attributes include rough, dull, and dry skin as well as droopy eyelids and dark eye circles [9]. These skin attributes that people associate with sleep deficiency are not merely limited to the cognitive characteristics.

Studies examining the effects of acute or chronic sleep deprivation on skin physiology report that individual's state of sleep can affect the health and physiology of skin, as well as their attractiveness. Studies reporting skin measurement results have shown that sleep deprivation can exacerbate several skin disorders [12]-[19]. Sleep deprivation increases activation of the sympathetic nervous system and activates the hypothalamic-pituitary-adrenal axis, leading to increased glucocorticoid secretion [14] [15] [20]. Increased production of glucocorticoids, which may affect the integrity of the lamellar bodies, impairs the integrity of the skin barrier [12] [16] [17] [18] [21]. Furthermore, a study in humans has revealed that 42 hours of acute sleep deprivation led to increased production of inflammatory cytokines including plasma interleukin- $1 \beta$ and tumor necrosis factor- $\alpha$, increased natural killer cell activity, and decreased skin barrier function recovery [18]. Deregulation of the immune system also impairs the integrity of collagen fibers [12] [18] [22]. These alterations might be related to various skin physiological properties, and it gives us clues that measuring the changed skin biophysical properties caused by sleep deprivation would be meaningful. Heretofore, scientific measuring of various skin attributes which might be regarded as the symptoms of sleep deficiency have rarely been conducted. This study was designed to assess the effects one night of sleep deprivation has on skin biophysical properties including transepidermal water loss (TEWL), facial pore size, and skin tone, hydration, elasticity, desquamation, translucency, and blood flow. 


\section{Methods}

This study was approved by the Institutional Review Board and all subjects provided written, informed consent prior to participation in the studies.

Twenty-four healthy Korean women aged 25 - 35 years $(28.3 \pm 2.9)$ participated in our study, which was conducted in October 2014 in the research center located in Seongnam, Korea. Subjects who were considered as having a normal sleep pattern according to diurnal type scale were included, and subjects who suffered from sleep disorders were excluded. To minimize the effects gender might have on skin biophysical properties after sleep deprivation [9] [23], only women were recruited for this study. We designed one group pretest-posttest comparing the effects of sleep deprivation [9] [10] [18] [24]. A night of normal sleep occurred in the subjects' own homes and sleep time was confirmed by sending messages to the research assistant. At least eight hours of sleep was required for a normal sleep as a habitual sleep pattern of each subject. The day after a normal sleep, the subjects visited a laboratory for baseline measurements and then they were allowed to leave the laboratory for their daily routines. Sleep during the daytime was not allowed and they had to return before dinner time. For a night of sleep deprivation, subjects were monitored and kept awake for one night (totally 24 hours of wakefulness) in a laboratory setting with controlled temperature $\left(24^{\circ} \mathrm{C} \pm 2^{\circ} \mathrm{C}\right)$ and relative humidity $(40 \% \pm 2 \%)$. The subjects were prohibited from exercise, caffeine, alcohol, and smoking 24 hours before and during the study, and their food intake during the sleep deprivation was standardized identically [18]. The skin condition of each subject was evaluated after normal sleep (baseline) and after sleep deprivation (24 hours of wakefulness). Measurements were conducted at the same time of each day in the morning, following the completion of facial cleansing and acclimation to the room condition. The skin of cheek, around eye, and lip areas were evaluated selectively for each parameter. Table 1 lists the parameters we evaluated and the equipment used to measure them, and they were measured in the order listed below.

Table 1. Measured skin biophysical properties and equipments used for measurements.

\begin{tabular}{ccc}
\hline Properties & Equipments & Measured areas \\
\hline Skin hydration & Corneometer ${ }^{\circledast}$ CM825 & Cheek, eye, lip \\
Transepidermal water loss (TEWL) & Tewameter ${ }^{\circledast}$ TM300 & Cheek, eye \\
Skin elasticity & Cutometer ${ }^{\oplus}$ MPA580 & Cheek, eye \\
Skin desquamation & Keratin sticker/i-Scope & Cheek \\
Translucency & Translucencymeter ${ }^{\circledast}$ TLS850 & Cheek \\
Facial pore & Visia-CR ${ }^{\infty}$ & Cheek \\
Skin tone & Visia-CR & Cheek, eye, lip \\
Skin blood flow & Laser Doppler Perfusion Imager & Eye, lip
\end{tabular}




\subsection{Skin Evaluation}

Skin hydration: A Corneometer ${ }^{\circledast}$ CM 825 (Courage \& Khazaka, Germany) was used to measure the water content of the cheek, lip, and the area around the eye. The instrument results are based on a capacitance measurement and expressed in arbitrary units (a.u.).

Trans Epidermal Water Loss (TEWL): A Tewameter ${ }^{\circledast}$ TM300 (Courage \& Khazaka, Germany) was used to measure TEWL of the cheek and the area around the eye. Measuring TEWL is an effective way to evaluate stratum corneum barrier function, and the results are expressed in $\mathrm{g} / \mathrm{m}^{2} \mathrm{~h}$.

Skin mechanical properties: A Cutometer ${ }^{\circledR}$ MPA 580 (Courage \& Khazaka, Germany) was used to measure the viscoelasticity of the cheek and the area around the eye. A probe with a $2 \mathrm{~mm}$ diameter and 450 mbar negative pressure were used for the measurement. The ratio of elastic recovery to the total deformation in the skin (R7 parameter, Ur/Uf), which represents the biological elasticity of the skin [25] was analyzed.

Skin desquamation: Dead skin cells were collected from the cheek with keratin stickers and then photographed using an i-Scope microscope (Moritex, Japan). Skin desquamation was quantified using an image analysis software (Image Pro Plus, USA).

Skin translucency: A Translucency Meter ${ }^{\circledR}$ TLS850 (Dia-Stron Ltd., UK) was used which measures reflected light from transmitted RGB LED light beam to the skin. We analyzed the Alpha, K, and Area values to determine cheek skin translucency. The Alpha value represents the rate of exponential attenuation of transmitted light, and the $\mathrm{K}$ value represents the amount of light that the probe detected. The Area value is obtained from the total amount of light scattered into the material. A decreased Alpha value and increased $\mathrm{K}$ and Area values indicated increased skin translucency.

Skin imaging: A VISIA-CR ${ }^{\circledast}$ (Canfield Scientific, Inc., USA) device was used to photograph each subject's frontal face image. The total area of pores in the cheek skin was calculated with an image analysis software (Image Pro Plus, USA). The skin lightness ( $\mathrm{L}^{*}$ value) of the cheek, lip, and infraorbital area was analyzed as well.

Skin blood flow: A Laser Doppler Perfusion Imager (Perimed, Sweden) was used to measure blood flow in the infraorbital and lip areas. Color-coded images of the blood flow were obtained as well.

\subsection{Statistical Analysis}

SPSS Statistics 21.0 (SPSS Inc., USA) was used for our statistical analysis. The Wilcoxon signed-rank (non-parametric) and paired (parametric) t-tests were used to compare the differences of skin parameters after sleep deprivation. $p$ value smaller than 0.05 was considered to be statistically significant.

\section{Results}

The results of the skin evaluations are presented in Table 2 and Figures 1-4. 
Table 2. Summary of skin biophysical parameters after sleep deprivation (mean \pm SEM).

\begin{tabular}{|c|c|c|c|}
\hline Properties & After normal sleep (baseline) & After sleep deprivation & p-value \\
\hline \multicolumn{4}{|l|}{ Skin hydration (a.u.) } \\
\hline Cheek & $65.53 \pm 2.15$ & $62.00 \pm 2.08^{\star *}$ & 0.003 \\
\hline Eye & $78.74 \pm 1.78$ & $77.00 \pm 1.33$ & 0.139 \\
\hline Lip & $56.67 \pm 1.45$ & $46.35 \pm 1.68^{\star * *}$ & 0.000 \\
\hline \multicolumn{4}{|l|}{$\begin{array}{c}\text { Transepidermal } \\
\text { water loss }\left(\mathrm{g} / \mathrm{m}^{2} \mathrm{~h}\right)\end{array}$} \\
\hline Cheek & $22.97 \pm 0.75$ & $23.79 \pm 0.73^{\star \star}$ & 0.002 \\
\hline Eye & $19.56 \pm 0.75$ & $20.33 \pm 0.80^{\star *}$ & 0.001 \\
\hline \multicolumn{4}{|l|}{ Elasticity (R7, a.u.) } \\
\hline Cheek & $0.343 \pm 0.007$ & $0.322 \pm 0.007^{\star * *}$ & 0.000 \\
\hline Eye & $0.365 \pm 0.013$ & $0.353 \pm 0.011^{*}$ & 0.033 \\
\hline \multicolumn{4}{|l|}{ Skin desquamation (pixel) } \\
\hline Cheek & $175550.83 \pm 3318.48$ & $180680.50 \pm 3034.80^{* *}$ & 0.001 \\
\hline \multicolumn{4}{|l|}{ Translucency (K, a.u.) } \\
\hline Cheek & $2356.46 \pm 66.88$ & $2262.90 \pm 57.91^{* *}$ & 0.003 \\
\hline \multicolumn{4}{|l|}{ Facial pore (pixel) } \\
\hline Cheek & $6173.54 \pm 567.66$ & $6713.00 \pm 609.68^{*}$ & 0.012 \\
\hline \multicolumn{4}{|l|}{ Skin tone ( $L^{*}$, a.u. $)$} \\
\hline Cheek & $70.72 \pm 0.60$ & $70.00 \pm 0.51^{\star}$ & 0.011 \\
\hline Eye (dark eye circle) & $65.70 \pm 0.94$ & $65.54 \pm 0.88$ & 0.332 \\
\hline Lip & $46.40 \pm 0.61$ & $46.30 \pm 0.66$ & 0.790 \\
\hline \multicolumn{4}{|l|}{ Skin blood flow (a.u.) } \\
\hline Eye & $0.89 \pm 0.04$ & $0.74 \pm 0.03^{* * *}$ & 0.000 \\
\hline Lip & $1.94 \pm 0.15$ & $1.78 \pm 0.11$ & 0.160 \\
\hline
\end{tabular}

Probability $\mathrm{p}\left({ }^{*} \mathrm{p}<0.05,{ }^{* *} \mathrm{p}<0.01,{ }^{* * *} \mathrm{p}<0.001\right)$.

There were statistically significant changes in multiple skin parameters after one night of sleep deprivation.

Skin hydration and skin barrier function: We found a significant decrease in the hydration of the cheek and lip skin. In particular, the decreasing rate of lip hydration was of greater magnitude. TEWL, which represents the integrity of the skin barrier, was significantly increased in the cheek and around the eye areas indicating that the barrier function was impaired (Table 2, Figure 1(a), Figure 1 (b)).

Skin biomechanical properties: The R7 (Ur/Uf) of the cheek and around the eye areas, which represent the biological elasticity of the skin [25], were decreased significantly after sleep deprivation (Table 2, Figure 1(c)). 


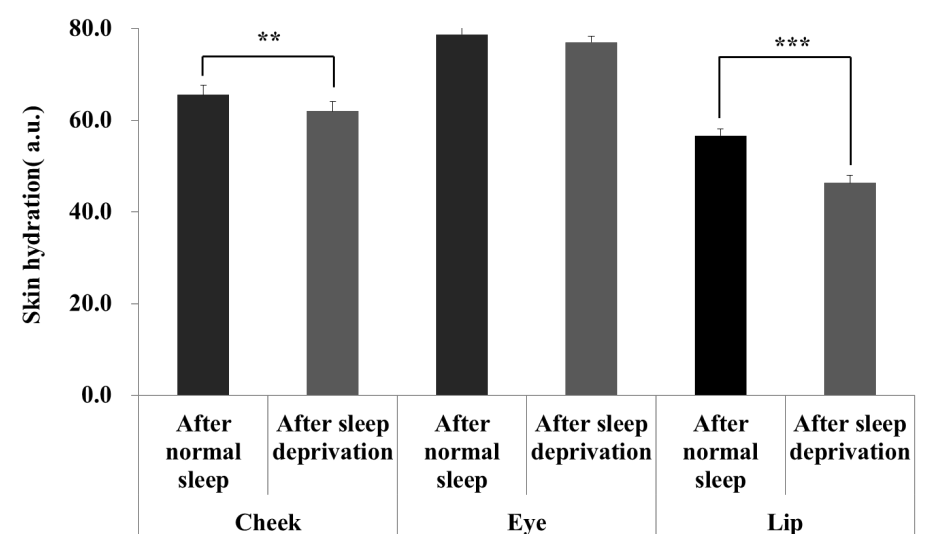

(a)

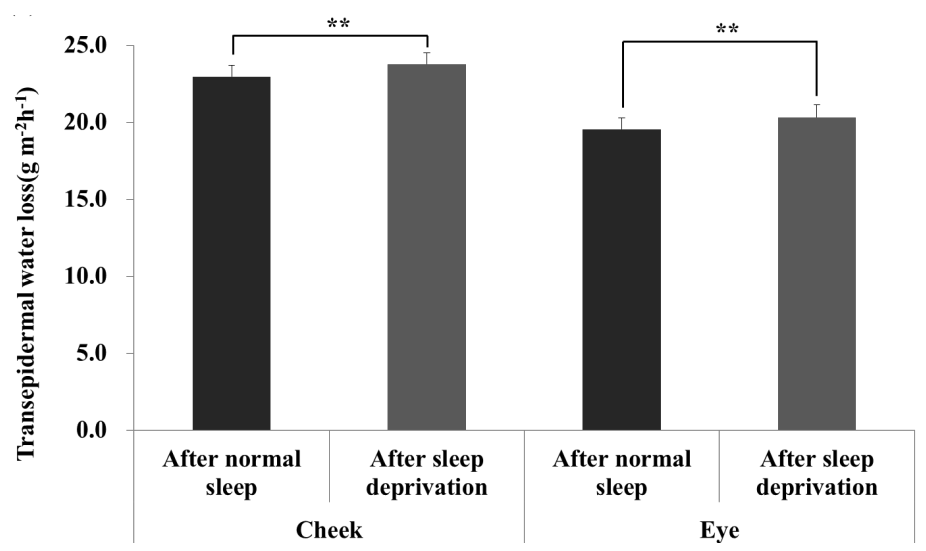

(b)

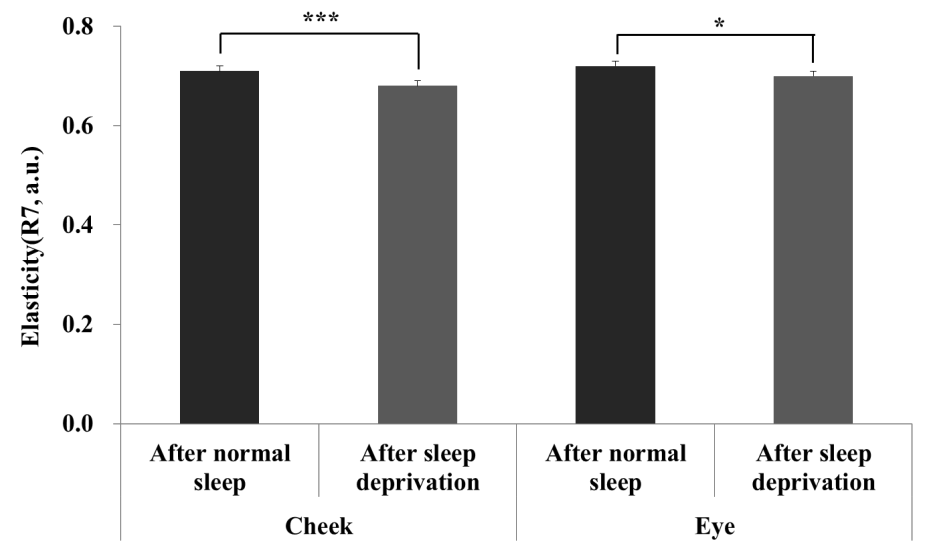

(c)

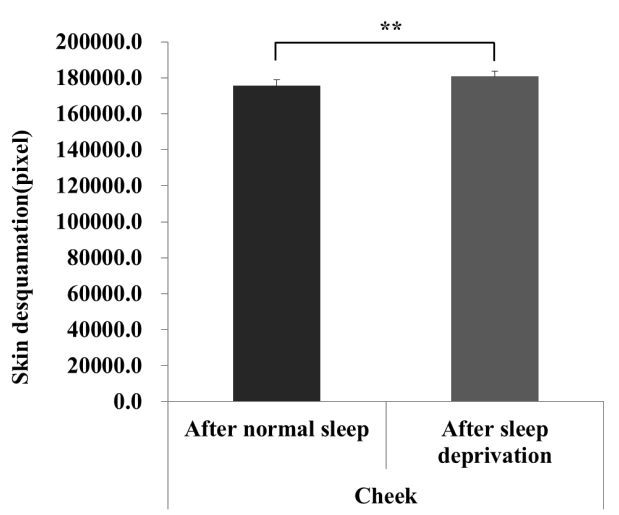

(d)

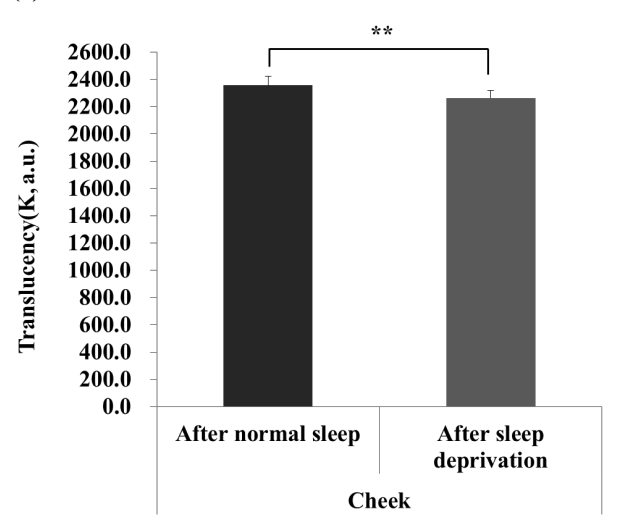

(e) 


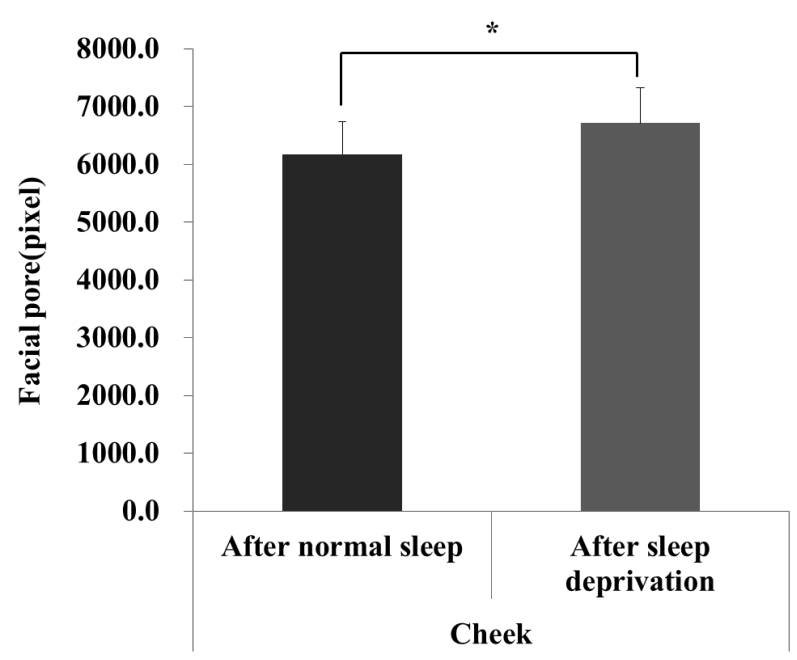

(f)

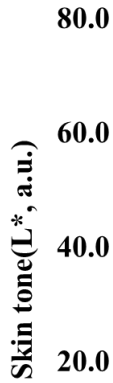

0.0
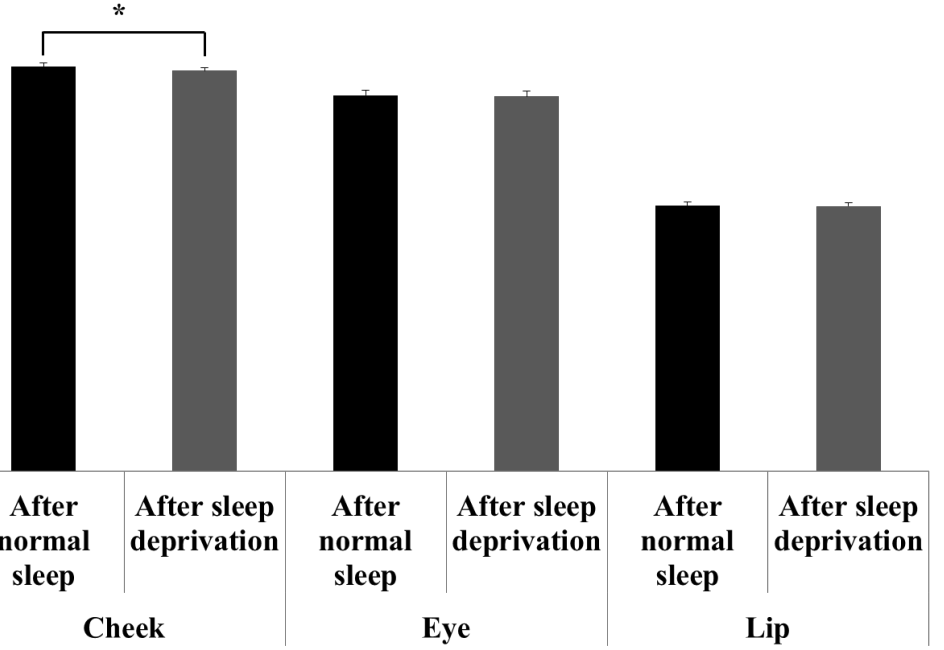

(g)

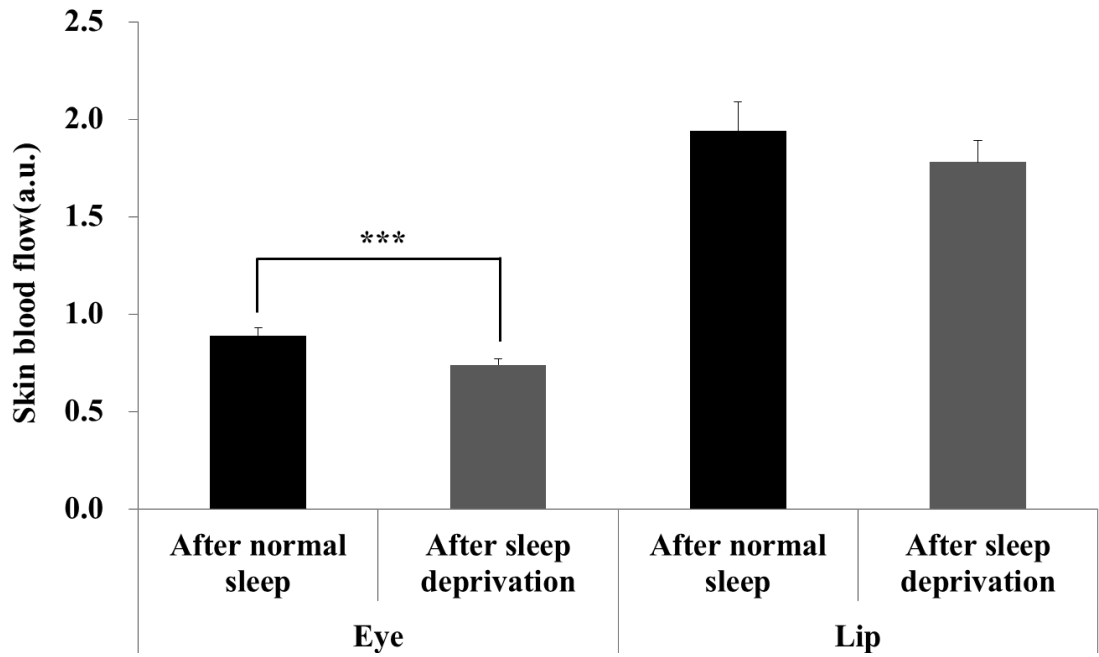

(h)

Figure 1. Skin biophysical parameters after sleep deprivation (mean \pm SEM). (a) Skin hydration; (b) Transepidermal water loss; (c) Skin elasticity; (d) Skin desquamation; (e) Skin translucency; (f) Facial pore; (g) Skin tone; (h) Skin blood flow. The graphs are represented for each measured area. Probability $\mathrm{p}\left({ }^{*} \mathrm{p}<0.05,{ }^{* *} \mathrm{p}<0.01,{ }^{* *} \mathrm{p}<0.001\right)$. 


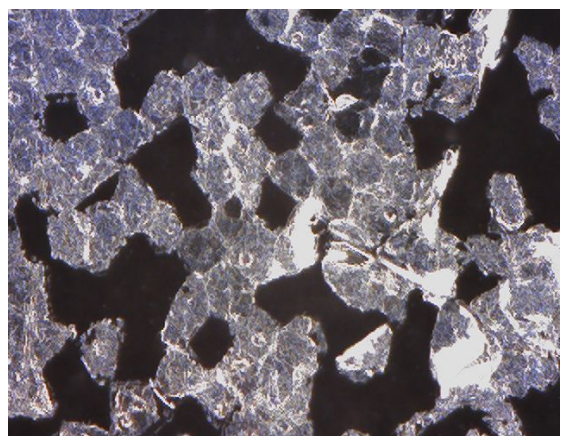

(a)

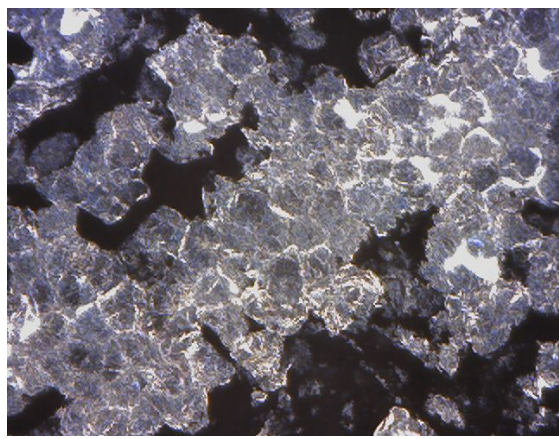

(b)

Figure 2. Representative images of skin desquamation in the cheek area (a) after normal sleep; (b) after sleep deprivation.

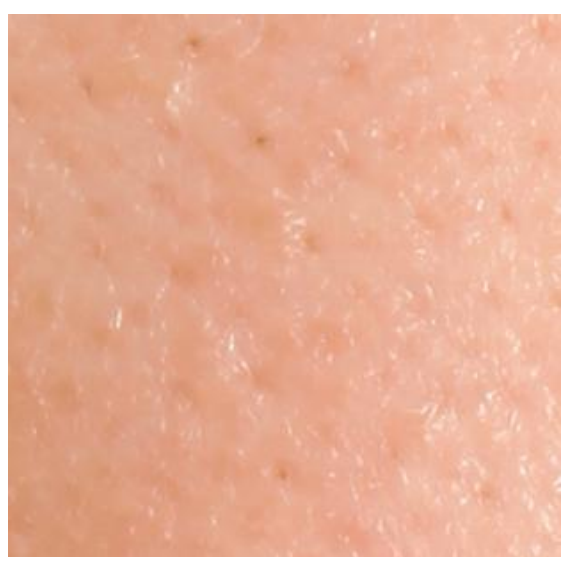

(a)

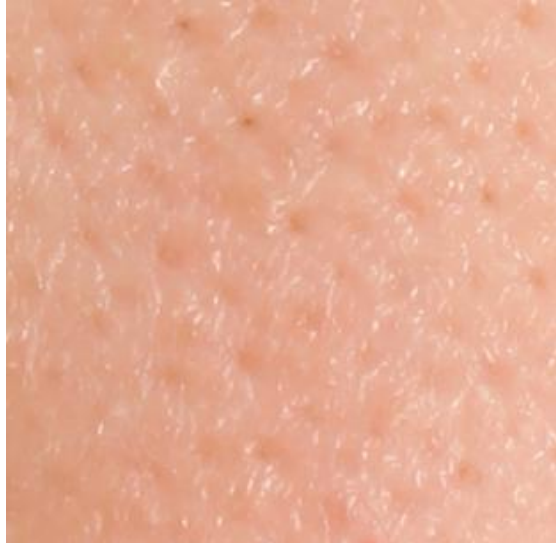

(b)

Figure 3. Representative images of skin pore in the cheek area (a) after normal sleep; (b) after sleep deprivation.

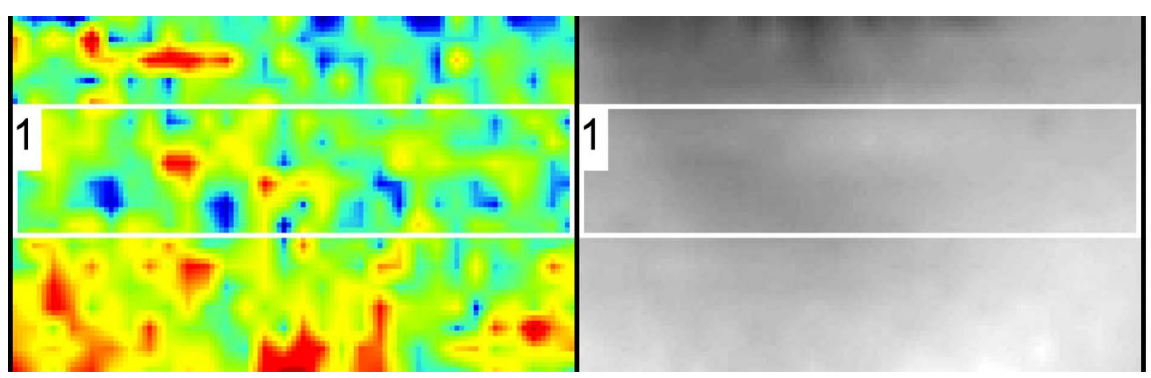

(a)

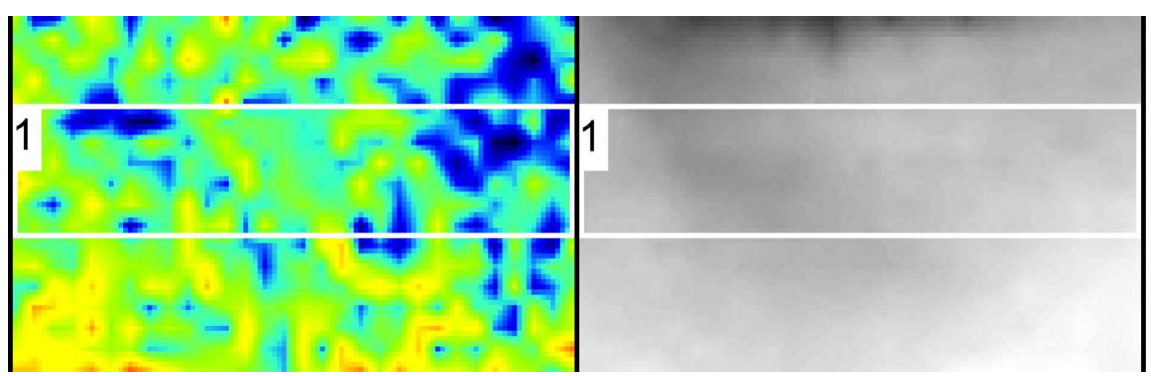

(b)

Figure 4. Representative images of skin blood flow in the infraorbital area (a) after normal sleep; (b) after sleep deprivation. 
Skin desquamation: The amounts of dead skin cells present in the cheek area were markedly increased after sleep deprivation (Table 2, Figure 1(d)). Taken images of keratin stickers also showed changed amount of dead skin cells (Figure 2).

Skin translucency: Skin translucency was significantly diminished after one night of sleep deprivation as indicated by decreased $\mathrm{K}$ values (Table 2, Figure 1(e)). There were also significantly increased Alpha values and decreased Area values, which provided further, indirect evidence of decreased skin translucency (data not shown).

Facial pores: There was a significant increase in the area of facial pores of the cheek following the period of sleep deprivation (Table 2, Figure 1(f)). The pores seemed to be enlarged and conspicuous as shown by VISIA-CR ${ }^{\circledast}$ imaging (Figure 3).

Skin tone: Skin lightness $\left(\mathrm{L}^{*}\right)$ as assessed by VISIA-CR ${ }^{\varpi}$ imaging was significantly decreased in the cheek. The $\mathrm{L}^{*}$ value of lip and infraorbital areas did not show a significant change (Table 2, Figure $1(\mathrm{~g})$ ).

Skin blood flow: Skin blood flow in the infraorbital area was significantly decreased, whereas the lip area showed a decrease in flow that did not reach statistical significance (Table 2, Figure 1(h)). Color-coded images of the eye area illustrated decreased blood flow after sleep deprivation by indicating perfusion from minimal (blue) to maximal (red) (Figure 4).

\section{Discussion}

We have verified that sleep deprivation affects various skin properties including TEWL, facial pore size, and skin tone, hydration, elasticity, desquamation, translucency, and blood flow. Multiple negative effects of sleep deprivation on the immune system, metabolism, and the skin have been reported [12]-[22] [24] [26] [27].

We confirmed that sleep deprivation leads to impaired skin barrier function as evidenced by increased TEWL even as skin hydration decreased. These findings are consistent with those of a previous study that showed a decreased skin barrier function after 42 hours of sleep deprivation [18]. Authors suggested that increased levels of plasma interleukin- $1 \beta$ and tumor necrosis factor- $\alpha$ resulting from stress may lead to impairment of the skin barrier function although the underlying mechanism for this increase in circulating cytokines was unclear. Similarly, Kahan et al. hypothesized that lack of sleep might impair skin integrity. They proposed that sleep deprivation causes increased glucocorticoid production, which contributes to the altered integrity of lamellar bodies, thus impairing skin integrity [12]. Deregulated immune system after sleep deprivation was also believed to affect the integrity of collagen fibers [12] [18] [22], which are the major constituent of the dermis responsible for skin elasticity. This altered integrity of collagen fibers is inadequate for explaining the decreased skin elasticity found in our study considering that the period of sleep deprivation was a shortterm and temporary. Instead, this change could be correlated with decreased 
skin hydration, as dry skin leads to a less soft and more rigid skin surface. Previous studies reported that epidermal hydration influences skin mechanical properties [28] [29] [30]. Dobrev observed a significant increase in delayed distension (Uv) and the viscoelastic-to-elastic ratio (Uv/Ue) as well as the net elasticity $(\mathrm{Ur} / \mathrm{Ue})$ and the ratio of elastic recovery to the total deformation (Ur/Uf) after epidermal hydration by moisturizers [30]. Therefore, the Cutometer ${ }^{\circledast}$ may underestimate skin elasticity in the presence of less hydrated skin because its measurement is based on a suction method. It would be worthwhile to study chronically sleep-deprived people to investigate the underlying mechanisms of altered skin elasticity.

The markedly increased skin scaling present after sleep deprivation also seems to be caused by decreased skin hydration. Skin desquamation increases as skin hydration decreases [31] [32] [33]. Cheek skin translucency was also decreased after the period of sleep deprivation. Measurement of skin translucency is based on the absorption and scattering of light. The translucency meter used in this study measures only internally scattered light, not light reflected from the surface. A previous study showed that skin translucency is influenced by skin hydration [34]. They proved that when the skin is moisturized, light shows less scattering within the stratum corneum and greater penetration into the deeper skin layers. Therefore, it could be that decreased hydration after sleep deprivation might contribute to decrease internal light scattering resulting in the decreased skin translucency we found. Another study reported that the dermal matrix, including organizations of fibers, affects the subsurface reflectance in the skin [35]. Based on this result, the dermal density of chronically sleep deprived people could be studied in the future to determine the correlation between skin translucency and the altered integrity of collagen fibers resulting from prolonged sleep deprivation.

Our analysis of facial photographs revealed that the pixels representing skin pores of the cheek were increased significantly. This could mean the facial pores were more conspicuous, rather than indicating an increased number of pores. Our hypothesis is that the substantially increased skin scaling resulting from sleep deprivation led to a coarse skin texture causing the skin pores to appear enlarged.

Skin tone changes are widely perceived as a symptom of sleep deficiency. In our study the lightness of cheek skin was decreased significantly whereas there was no significant change in the lightness of the lip. Dark circles under the eyes are a primary feature indicating fatigue and stress [9], however, we found no statistically significant change in the $L^{\star}$ value under the eyes although there was a trend toward lower values. Blood flow stagnation contributes significantly to the development of dark circles beneath the eyes [36], and this could account for the decreased skin blood flow that we found under the eyes. Our skin blood flow results could be related to other studies as well that showed increase in skin blood flow during sleep [37]. The mechanisms of increased blood flow during sleep is unclear, however, it is associated with barrier defense of the skin as well 
as energy metabolism [38]. It would be meaningful to study the relation between the impaired barrier defense and the decreased skin blood flow induced by sleep deprivation in the future.

Generally, people perceive their skin to be dry, rough, dull, as well as droopy eyelids and dark circles under the eyes after a sleepless night. In this study, the results proved that the skin features that are recognized as symptoms of sleep deficiency actually showed significant changes, and some of these changes were confirmed in the eye, lip, as well as the cheek areas. These findings underline that the affected facial regions are crucial in the social communication [9] [11]. The altered features include decreased skin hydration and this affected most of the other skin physiological properties we examined. Furthermore, we noted changes in the optical properties concerning the light reflected from the skin, such as altered skin translucency and coarse skin texture.

Further studies that compare the sex-based differences in skin biophysical properties after sleep deprivation would be worthwhile. A previous study regarding the visual features of sleep deprivation showed that only men were perceived as being paler and having more tense lips when sleep deprived, suggesting sex-based differences in the response to sleep deprivation [9]. One night of sleep deprivation might be quite stressful to the participants in this study, therefore, the skin biophysical changes observed could be influenced by the stress induced by the method as well [12] [39]. Further studies are needed to figure out the effects only sleep deprivation has on the skin biophysical properties by setting the control group under the other stressful situations or under the same experimental laboratory with habitual sleeping conditions.

Even though the skin biophysical changes induced by sleep deprivation in this study were mild, we hypothesize that chronic repetition of sleep loss could have more serious implications. Therefore, studies examining the correlation between prolonged sleep deprivation or chronic sleep deficiency and skin physiology remain to be explored.

In modern society, many people suffer from a lack of sleep and are highly interested in their appearance. We identified a scientific relationship between sleep and skin physiology, which might become a cornerstone of related study field. Furthermore, solutions for reducing skin changes after sleep deprivation could be presented. Cosmetics to increase lip hydration or products for covering skin optical defects would be beneficial.

\section{Acknowledgements}

This research was supported by AMOREPACIFIC Corporation.

\section{References}

[1] Knutson, K.L., Spiegel, K., Penev, P. and Van Cauter, E. (2007) The Metabolic Consequences of Sleep Deprivation. Sleep Medicine Reviews, 11, 163-178. https://doi.org/10.1016/j.smrv.2007.01.002

[2] Pilcher, J.J. and Huffcutt, A.J. (1996) Effects of Sleep Deprivation on Performance: 
A Meta-Analysis. Sleep, 19, 318-326.

[3] Drummond, S.P., Brown, G.G., Gillin, J.C., et al. (2000) Altered Brain Response to Verbal Learning Following Sleep Deprivation. Nature, 403, 655-657.

[4] Ferrara, M. and De Gennaro, L. (2001) How Much Sleep Do We Need? Sleep Medicine Reviews, 5, 155-179. https://doi.org/10.1053/smrv.2000.0138

[5] Alvarez, G.G. and Ayas, N.T. (2004) The Impact of Daily Sleep Duration on Health: A Review of the Literature. Progress in Cardiovascular Nursing, 19, 56-59. https://doi.org/10.1111/j.0889-7204.2004.02422.x

[6] Lange, T., Dimitrov, S. and Born, J. (2010) Effects of Sleep and Circadian Rhythm on the Human Immune System. Annals of the New York Academy of Sciences, 1193, 48-59. https://doi.org/10.1111/j.1749-6632.2009.05300.x

[7] Majde, J.A. and Krueger, J.M. (2005) Links between the Innate Immune System and Sleep. Journal of Allergy and Clinical Immunology, 116, 1188-1198. https://doi.org/10.1016/j.jaci.2005.08.005

[8] Rajaratnam, S.M. and Arendt, J. (2001) Health in a 24-h Society. The Lancet, 358, 999-1005. https://doi.org/10.1016/S0140-6736(01)06108-6

[9] Sundelin, T., Lekander, M., Kecklund, G., et al. (2013) Cues of Fatigue: Effects of Sleep Deprivation on Facial Appearance. Sleep, 36, 1355-1360.

https://doi.org/10.5665/sleep.2964

[10] Axelsson, J., Sundelin, T., Ingre, M., et al. (2010) Beauty Sleep: Experimental Study on the Perceived Health and Attractiveness of Sleep Deprived People. BMJ, 341, c6614. https://doi.org/10.1136/bmj.c6614

[11] Haxby, J.V., Hoffman, E.A. and Gobbini, M.I. (2000) The Distributed Human Neural System for Face Perception. Trends in Cognitive Sciences, 4, 223-233. https://doi.org/10.1016/S1364-6613(00)01482-0

[12] Kahan, V., Andersen, M.L., Tomimori, J. and Tufik, S. (2010) Can Poor Sleep Affect Skin Integrity? Medical Hypotheses, 75, 535-537.

https://doi.org/10.1016/j.mehy.2010.07.018

[13] Mostaghimi, L., Obermeyer, W.H., Ballamudi, B., Martinez-Gonzalez, D. and Benca, R.M. (2005) Effects of Sleep Deprivation on Wound Healing. Journal of Sleep Research, 14, 213-219. https://doi.org/10.1111/j.1365-2869.2005.00455.x

[14] Irwin, M., Thompson, J., Miller, C., Gillin, J.C. and Ziegler, M. (1999) Effects of Sleep and Sleep Deprivation on Catecholamine and Interleukin-2 Levels in $\mathrm{Hu}$ mans: Clinical Implications. The Journal of Clinical Endocrinology \& Metabolism, 84, 1979-1985. https://doi.org/10.1210/jc.84.6.1979

[15] Spiegel, K., Leproult, R. and Van Cauter, E. (1999) Impact of Sleep Debt on Metabolic and Endocrine Function. The Lancet, 354, 1435-1439. https://doi.org/10.1016/S0140-6736(99)01376-8

[16] Denda, M., Tsuchiya, T., Elias, P.M. and Feingold, K.R. (2000) Stress Alters Cutaneous Permeability Barrier Homeostasis. American Journal of Physiology. Regulatory, Integrative and Comparative Physiology, 278, R367-R372.

[17] Kao, J.S., Fluhr, J.W., Man, M.Q., et al. (2003) Short-Term Glucocorticoid Treatment Compromises Both Permeability Barrier Homeostasis and Stratum Corneum Integrity: Inhibition of Epidermal Lipid Synthesis Accounts for Functional Abnormalities. Journal of Investigative Dermatology, 120, 456-464. https://doi.org/10.1046/j.1523-1747.2003.12053.x

[18] Altemus, M., Rao, B., Dhabhar, F.S., Ding, W. and Granstein, R.D. (2001) StressInduced Changes in Skin Barrier Function in Healthy Women. Journal of Investigative Dermatology, 117, 309-317. https://doi.org/10.1046/j.1523-1747.2001.01373.x 
[19] Kushida, C.A., Everson, C.A., Suthipinittharm, P., et al. (1989) Sleep Deprivation in the Rat: VI. Skin Changes. Sleep, 12, 42-46.

[20] Meerlo, P., Koehl, M., Van der Borght, K. and Turek, F.W. (2002) Sleep Restriction Alters the Hypothalamic-Pituitary-Adrenal Response to Stress. Journal of Neuroendocrinology, 14, 397-402. https://doi.org/10.1046/j.0007-1331.2002.00790.x

[21] Garg, A., Chren, M.M., Sands, L.P., et al. (2001) Psychological Stress Perturbs Epidermal Permeability Barrier Homeostasis: Implications for the Pathogenesis of Stress-Associated Skin Disorders. Archives of Dermatology, 137, 53-59. https://doi.org/10.1001/archderm.137.1.53

[22] Kahan, V., Andersen, M.L., Tomimori, J. and Tufik, S. (2009) Stress, Immunity and Skin Collagen Integrity: Evidence from Animal Models and Clinical Conditions. Brain, Behavior, and Immunity, 23, 1089-1095.

https://doi.org/10.1016/j.bbi.2009.06.002

[23] Kirschbaum, C., Kudielka, B.M., Gaab, J., Schommer, N.C. and Hellhammer, D.H. (1999) Impact of Gender, Menstrual Cycle Phase, and Oral Contraceptives on the Activity of the Hypothalamus-Pituitary-Adrenal Axis. Psychosomatic Medicine, 61, 154-162. https://doi.org/10.1097/00006842-199903000-00006

[24] Sauvet, F., Leftheriotis, G., Gomez-Merino, D., et al. (2010) Effect of Acute Sleep Deprivation on Vascular Function in Healthy Subjects. Journal of Applied Physiology, 108, 68-75. https://doi.org/10.1152/japplphysiol.00851.2009

[25] Dobrev, H.P. (2002) A Study of Human Skin Mechanical Properties by Means of Cutometer. Folia Medica, 44, 5-10.

[26] Everson, C.A. (2005) Clinical Assessment of Blood Leukocytes, Serum Cytokines, and Serum Immunoglobulins as Responses to Sleep Deprivation in Laboratory Rats. American Journal of Physiology. Regulatory, Integrative and Comparative Physiology, 289, R1054-R1063. https://doi.org/10.1152/ajpregu.00021.2005

[27] Mullington, J.M., Haack, M., Toth, M., Serrador, J.M. and Meier-Ewert, H.K. (2009) Cardiovascular, Inflammatory, and Metabolic Consequences of Sleep Deprivation. Progress in Cardiovascular Diseases, 51, 294-302. https://doi.org/10.1016/j.pcad.2008.10.003

[28] Jemec, G.B., Jemec, B., Jemec, B.I. and Serup, J. (1990) The Effect of Superficial Hydration on the Mechanical Properties of Human Skin in Vivo: Implications for Plastic Surgery. Plastic and Reconstructive Surgery, 85, 100-103. https://doi.org/10.1097/00006534-199001000-00017

[29] Auriol, F., Vaillant, L., Machet, L., Diridollou, S. and Lorette, G. (1993) Effects of Short-Time Hydration on Skin Extensibility. Acta Dermato Venereologica, 73, 344347.

[30] Dobrev, H. (2000) Use of Cutometer to Assess Epidermal Hydration. Skin Research and Technology, 6, 239-244. https://doi.org/10.1034/j.1600-0846.2000.006004239.x

[31] Nam, G.W., Baek, J.H., Koh, J.S. and Hwang, J.K. (2015) The Seasonal Variation in Skin Hydration, Sebum, Scaliness, Brightness and Elasticity in Korean Females. Skin Research and Technology, 21, 1-8. https://doi.org/10.1111/srt.12145

[32] Jemec, G.B. and Serup, J. (1991) Scaling, Dry Skin and Gender. A Bioengineering Study of Dry Skin. Acta Dermato Venereologica, 177, 26-28.

[33] Proksch, E. and Lachapelle, J.M. (2005) The Management of Dry Skin with Topical Emollients-Recent Perspectives. Journal der Deutschen Dermatologischen Gesellschaft, 3, 768-774. https://doi.org/10.1111/j.1610-0387.2005.05068.x

[34] Jiang, Z.X. and DeLaCruz, J. (2011) Appearance Benefits of Skin Moisturization. Skin Research and Technology, 17, 51-55. 
https://doi.org/10.1111/j.1600-0846.2010.00462.x

[35] Kim, H.J., Baek, J.H., Eo, J.E., et al. (2015) Dermal Matrix Affects Translucency of Incident Light on the Skin. Skin Research and Technology, 21, 41-46.

https://doi.org/10.15323/techart.2015.02.2.1.41

[36] Masuda, Y., Takahashi, M., Satou, A., et al. (2004) Dermatological Study on Dark Eye Circles and Their Treatment with Newly Developed Cosmetics. International Journal of Cosmetic Science, 26, 315-315. https://doi.org/10.1111/j.1467-2494.2004.00242_2.x

[37] Van Someren, E.J. (2004) Sleep Propensity Is Modulated by Circadian and Behavior-Induced Changes in Cutaneous Temperature. Journal of Thermal Biology, 29, 437-444. https://doi.org/10.1016/j.jtherbio.2004.08.003

[38] Van Someren, E.J. (2006) Mechanisms and Functions of Coupling between Sleep and Temperature Rhythms. Progress in Brain Research, 153, 309-324. https://doi.org/10.1016/S0079-6123(06)53018-3

[39] Papale, L.A., Andersen, M.L., Antunes, I.B., Alvarenga, T.A.F. and Tufik, S. (2005) Sleep Pattern in Rats under Different Stress Modalities. Brain Research, 1060, $47-$ 54. https://doi.org/10.1016/j.brainres.2005.08.021

Submit or recommend next manuscript to SCIRP and we will provide best service for you:

Accepting pre-submission inquiries through Email, Facebook, LinkedIn, Twitter, etc. A wide selection of journals (inclusive of 9 subjects, more than 200 journals) Providing 24-hour high-quality service

User-friendly online submission system Fair and swift peer-review system Efficient typesetting and proofreading procedure Display of the result of downloads and visits, as well as the number of cited articles Maximum dissemination of your research work

Submit your manuscript at: http://papersubmission.scirp.org/

Or contact jcdsa@scirp.org 